\title{
On Controllability for Linear Differential-Algebraic Control Systems Using Equivalent Form
}

\author{
ABED MAKRELOUFI ${ }^{1}$, MOHAMMED BENHARRAT ${ }^{2 *}$ \\ Department of Systems Engineering (Ex. Department of Mathematics and informatics) \\ National Polytechnic School of Oran-Maurice Audin (Ex. ENSET of Oran) \\ BP 1523 Oran-El M'naouar, 31000 Oran \\ ALGERIA
}

\begin{abstract}
We investigate different concepts related to the controllability of linear constant coefficient differentialalgebraic equations. Concepts like impulse controllability, controllability at infinity, strong and complete controllability are described and defined by using an equivalent form for linear time-invariant singular systems. Some real-life problems are given to illustrate the presented theory.
\end{abstract}

Key-Words: Differential-Algebraic Equations, Controllability, Equivalent Form.

Received: March 5, 2021. Revised: September 14, 2021. Accepted: September 24, 2021. Published: September 30, 2021.

\section{Introduction}

Many physical phenomena are modeled by differential equations, but sometimes this mathematical model contains algebraic equations. Systems containing two types of differential and algebraic equations are called differential-algebraic equations (DAEs) [also known as singular systems, implicit systems, descriptor systems]. These systems have become a model of a wide variety of problems in economics, demography, mechanical systems [11], the simulation of the dynamics of multi-body systems [18], [19], electrical networks [8], [20], fluid mechanics [22] and chemistry [17], which often cannot be modeled by standard ODE systems.

In this paper, we consider linear differentialalgebraic control systems of the form

$$
E \dot{x}(t)=A x(t)+B u(t)
$$

where $E, A \in \mathbb{R}^{m \times n}, B \in \mathbb{R}^{m \times r}$ and $\operatorname{rank} E=r_{0} \leq$ $\min \{m, n\}$. The unknown $x(t) \in \mathbb{R}^{n}$ is the dynamic state and $u(t) \in \mathbb{R}^{r}$ is the control. The system (11) will be a classic linear system if $E$ and $A$ are are square matrices and $E=I$. If $m=n$ and there exists a $\lambda \in \mathbb{C}$ such that the matrix pencil $(\lambda E-A)$ is invertible, then the system (11) is called regular descriptor system; otherwise, it is called irregular descriptor system. Note that rectangular descriptor systems are irregular descriptor systems.

A trajectory $(x, u): \mathbb{R} \longrightarrow \mathbb{R}^{n} \times \mathbb{R}^{r}$ is said to be a solution of (11) if, and only if, it belongs to the behav- ior:

$$
\begin{aligned}
\mathfrak{B}_{[E, A, B]}:= & \left\{(x, u) \in W_{\text {loc }}^{1,1}\left(\mathbb{R}, \mathbb{R}^{n}\right) \times L_{\text {loc }}^{1}\left(\mathbb{R}, \mathbb{R}^{r}\right):\right. \\
& (x, u) \text { satisfies }(\mathbb{\square}) \text { for almost all } \\
& t \in \mathbb{R}\},
\end{aligned}
$$

where

$$
L_{l o c}^{1}\left(\mathbb{R}, \mathbb{R}^{r}\right):=\text { Locally Lebesgue integrable func- }
$$
tions $u: \mathbb{R} \longrightarrow \mathbb{R}^{r}$, and

$W_{\text {loc }}^{1,1}\left(\mathbb{R}, \mathbb{R}^{n}\right):=\left\{x: \mathbb{R} \longrightarrow \mathbb{R}^{n}: x, \dot{x} \in L_{\text {loc }}^{1}\left(\mathbb{R}, \mathbb{R}^{n}\right)\right\}$

Contrary to the theory of controllability of ordinary systems, there are various concepts of controllability for DAEs: C-controllability, R-controllability, I-controllability, infinite controllability, ..., etc. These concepts were well established first in the work of Luenberger (1978) [12], Verghese et al. (1979) [21], Pandolfi (1980) [16], Campbell (1980) [3], Yip and Sincovec (1981) [23], Cobb (1984) [6], Christodoulou and Paraskevopoulos (1985) [4] , and then studied for various applications in the design of control systems in the works of Dai (1989) [5], Hou and Müller (1995) [10], Darouach and Boutayeb (1995) [7], Hou (2004 ) [9], Zhang et al. (2012) [24], Mishra et al. (2016) [15] and its references.

The paper is organized as follows. In Section 2 , we present the basic theoretic results for the controllability of singular systems, and we give an equivalent form of these systems. In Section 3, by using an equivalent form for linear time-invariant singular systems, we give necessary and sufficient conditions for impulse controllability, controllability at infinity, strong and complete controllability. We prove that Icontrollability for these system is equivalent to the 
controllability at infinity for another descriptor system obtained by an equivalent form for a singular system. Finally, in Section $\rrbracket$, some real-life examples are also provided for illustrating our ideas.

\section{Preliminary results}

In this section, we will present some findings regarding controllability for descriptor systems which we will need in the next sections. All these results are extracted from [1], [14] and [15].

Definition 1. The system (11) is called controllable at infinity if and only if

$$
\forall x_{0} \in \mathbb{R}^{n}, \exists(x, u) \in \mathfrak{B}_{[E, A, B]}: x(0)=x_{0} .
$$

The system (11) is controllable at infinity if and only if $\nu=\mathbb{R}^{n}$, where $\nu$ is the set of all consistent initial conditions for the system (11) and is defined as follows

$$
\nu=\left\{x_{0} \in \mathbb{R}^{n}, \exists(x, u) \in \mathfrak{B}_{[E, A, B]}: x(0)=x_{0}\right\} .
$$

Definition 2. The system (11) is called impulse controllable (I-controllable) if and only if

$$
\forall x_{0} \in \mathbb{R}^{n}, \exists(x, u) \in \mathfrak{B}_{[E, A, B]}: E x(0)=E x_{0} .
$$

The system (11) is I-controllable if and only if $\nu_{\omega}=$ $\mathbb{R}^{n}$, where $\nu_{\omega}=\mathbb{R}^{n}$ is the set of all weakly consistent initial conditions for the system (11) and is defined as follows

$\nu=\left\{x_{0} \in \mathbb{R}^{n}, \exists(x, u) \in \mathfrak{B}_{[E, A, B]}: E x(0)=E x_{0}\right\}$.

Definition 3. The system (11) is called completely controllable (C-controllable) if and only if

$$
\begin{gathered}
\exists T>0, \forall x_{0}, x_{f} \in \mathbb{R}^{n}, \exists(x, u) \in \mathfrak{B}_{[E, A, B]}: \\
x(0)=x_{0} \quad \text { and } \quad x(T)=x_{f} .
\end{gathered}
$$

Definition 4. The system (11) is called strongly controllable (S-controllable) if and only if

$$
\begin{gathered}
\exists T>0, \forall x_{0}, x_{f} \in \mathbb{R}^{n}, \exists(x, u) \in \mathfrak{B}_{[E, A, B]}: \\
E x(0)=E x_{0} \quad \text { and } \quad E x(T)=E x_{f} .
\end{gathered}
$$

Proposition 1. The system (Z) is controllable at infinity if and only if

$$
\operatorname{rank}[E, B]=\operatorname{rank}[E, A, B]
$$

Proposition 2. The system ( (ل) is $C$-controllable if and only if condition (2) is satisfied together with

$$
\operatorname{rank}[\lambda E-A, B]=\operatorname{rank}[E, A, B], \quad \forall \lambda \in \mathbb{C} .
$$

Proposition 3. The system (7) is I-controllable if and only if

$$
\operatorname{rank}\left[\begin{array}{ccc}
E & 0 & 0 \\
A & E & B
\end{array}\right]=\operatorname{rank}[E, A, B]+\operatorname{rank} E .
$$

Proposition 4. The system (I]) is $S$-controllable if and only if both the conditions (3) and (4) are satisfied.

Remark 1. For regular descriptor systems, the above Propositions can be found in Dai (1989b) where $\operatorname{rank}[E, A, B]$ is replaced by $n$ which is the order of the matrix $E$ or $A$.

Remark 2. We have the following implications:

$$
\begin{array}{ccc}
\text { C-controllability } & \Longrightarrow & \text { S-controllability } \\
\Downarrow & \Downarrow \\
\text { Controllability at infinity } & \Longrightarrow & \text { I-controllability }
\end{array}
$$

Now, we're going to give an equivalent form of a singular system (11). For that, we assume that $\operatorname{rank}(E)=r_{0} \leq \min \{m, n\}$. From matrix theory, we know that there exist nonsingular matrices $P \in \mathbb{R}^{m \times m}$ and $Q \in \mathbb{R}^{n \times n}$ such that

$$
P E Q=\left[\begin{array}{cc}
I_{r_{0}} & 0 \\
0 & 0
\end{array}\right]
$$

By taking the coordinate transformation

$$
x=Q\left(\begin{array}{l}
x_{1} \\
x_{2}
\end{array}\right), x_{1} \in \mathbb{R}^{r_{0}}, x_{2} \in \mathbb{R}^{n-r_{0}},
$$

system (11) is equivalent to

$$
(E F)\left\{\begin{array}{l}
\dot{x}_{1}(t)=A_{11} x_{1}(t)+A_{12} x_{2}+B_{1} u(t) \\
0=A_{21} x_{1}(t)+A_{22} x_{2}+B_{2} u(t)
\end{array}\right.
$$

where

$$
P A Q=\left[\begin{array}{ll}
A_{11} & A_{12} \\
A_{21} & A_{22}
\end{array}\right], P B=\left[\begin{array}{l}
B_{1} \\
B_{2}
\end{array}\right] .
$$

where $A_{11} \in \mathbb{R}^{r_{0} \times r_{0}}, A_{12} \in \mathbb{R}^{r_{0} \times\left(n-r_{0}\right)}, A_{21} \in$ $\mathbb{R}^{\left(m-r_{0}\right) \times r_{0}}, A_{22} \in \mathbb{R}^{\left(m-r_{0}\right) \times\left(n-r_{0}\right)}, B_{1} \in \mathbb{R}^{r_{0} \times r}$ and $B_{2} \in \mathbb{R}^{\left(m-r_{0}\right) \times r}$. The system (6) is an equivalent form $(E F)$ of (11), and it clearly reflects the physical meaning of the singular systems. In this transformation, matrices $U$ and $V$ are not unique. The first equation in (6) is a differential one composed of dynamic subsystems, and the second equation is an algebraic equation that represents the connection between subsystems. Thus, singular systems may be viewed as composite systems formed by several interconnected subsystems. Furthermore, sub-states $x_{1}$ and $x_{2}$ reflect a layer property in some singular systems: one layer has a dynamic property (described by the differential equation); the other has an interconnection, constraint, and administration properties (described by the algebraic equation). 


\section{Main results}

Theorem 1. The system (11) is controllable at infinity if and only if the system (6) satisfies

$$
\operatorname{rank} B_{2}=\operatorname{rank}\left[\begin{array}{lll}
A_{21} & A_{22} & B_{2}
\end{array}\right] .
$$

Proof. The controllability at infinity for the system (11) is equivalent to

$$
\operatorname{rank}[E, B]=\operatorname{rank}[E, A, B],
$$

then

$$
\begin{aligned}
& \operatorname{rank}\left(P\left[\begin{array}{ll}
E & B
\end{array}\right]\left[\begin{array}{cc}
Q & 0 \\
0 & I_{r}
\end{array}\right]\right)= \\
& \operatorname{rank}\left(P\left[\begin{array}{lll}
E & A & B
\end{array}\right]\left[\begin{array}{ccc}
Q & 0 & 0 \\
0 & Q & 0 \\
0 & 0 & I_{r}
\end{array}\right]\right) .
\end{aligned}
$$

this is equivalent to

$$
\operatorname{rank}\left[\begin{array}{ll}
P E Q & P B
\end{array}\right]=\operatorname{rank}[P E Q \quad P A Q \quad P B],
$$

applying the decomposition (5) and (7), the above condition is equivalent to

$$
\begin{aligned}
\operatorname{rank} & {\left[\begin{array}{ccc}
I_{r_{0}} & 0 & B_{1} \\
0 & 0 & B_{2}
\end{array}\right] } \\
& =\operatorname{rank}\left[\begin{array}{ccccc}
I_{r_{0}} & 0 & A_{11} & A_{12} & B_{1} \\
0 & 0 & A_{21} & A_{22} & B_{2}
\end{array}\right],
\end{aligned}
$$

then

$$
r_{0}+\operatorname{rank} B_{2}=r_{0}+\operatorname{rank}\left[\begin{array}{lll}
A_{21} & A_{22} & B_{2}
\end{array}\right],
$$

therefore

$$
\operatorname{rank} B_{2}=\operatorname{rank}\left[\begin{array}{lll}
A_{21} & A_{22} & B_{2}
\end{array}\right] .
$$

Theorem 2. The system (11) is I-controllable if and only if

$$
\operatorname{rank}\left[\begin{array}{ll}
A_{22} & B_{2}
\end{array}\right]=\operatorname{rank}\left[\begin{array}{lll}
A_{21} & A_{22} & B_{2}
\end{array}\right] .
$$

Proof. From (4), I-controllability for the system (1) is equivalent to

$$
\begin{gathered}
\operatorname{rank}\left(\left[\begin{array}{cc}
P & 0 \\
0 & P
\end{array}\right]\left[\begin{array}{ccc}
E & 0 & 0 \\
A & E & B
\end{array}\right]\left[\begin{array}{ccc}
Q & 0 & 0 \\
0 & Q & 0 \\
0 & 0 & I_{r}
\end{array}\right]\right)= \\
\operatorname{rank}\left(P[E, A, B]\left[\begin{array}{ccc}
Q & 0 & 0 \\
0 & Q & 0 \\
0 & 0 & I_{r}
\end{array}\right]\right)+\operatorname{rank} E .
\end{gathered}
$$

Then

$$
\begin{aligned}
\operatorname{rank} & {\left[\begin{array}{ccccc}
I_{r_{0}} & 0 & 0 & 0 & 0 \\
0 & 0 & 0 & 0 & 0 \\
A_{11} & A_{12} & I_{r_{0}} & 0 & B_{1} \\
A_{21} & A_{22} & 0 & 0 & B_{2}
\end{array}\right]=} \\
& \operatorname{rank}\left[\begin{array}{ccccc}
I_{r_{0}} & 0 & A_{11} & A_{12} & B_{1} \\
0 & 0 & A_{21} & A_{22} & B_{2}
\end{array}\right]+\text { rankE. }
\end{aligned}
$$

$$
\begin{aligned}
& \text { Therefore } \\
& 2 r_{0}+\operatorname{rank}\left[\begin{array}{ll}
A_{22} & B_{2}
\end{array}\right]= \\
& r_{0}+\operatorname{rank}\left[\begin{array}{lll}
A_{21} & A_{22} & B_{2}
\end{array}\right]+\operatorname{rankE.}
\end{aligned}
$$$$
\text { This is equivalent to }
$$

$$
\operatorname{rank}\left[\begin{array}{ll}
A_{22} & B_{2}
\end{array}\right]=\operatorname{rank}\left[\begin{array}{lll}
A_{21} & A_{22} & B_{2}
\end{array}\right] .
$$

Hence, the theorem is proved.

Theorem 3. The system (11) is C-controllable if and only if condition (8) is satisfied and

$$
\operatorname{rank}\left[\lambda I_{r_{0}}-A_{11}-A_{12} \quad B_{1}\right]=r_{0}, \quad \forall \lambda \in \mathbb{C} .
$$

Proof. From (3), C-controllability for the system (11) is equivalent to

$$
\begin{aligned}
\operatorname{rank} & \left(P\left[\begin{array}{lll}
E & A & B
\end{array}\right]\left[\begin{array}{ccc}
Q & 0 & 0 \\
0 & Q & 0 \\
0 & 0 & I_{r}
\end{array}\right]\right) \\
& =\operatorname{rank}\left(P[\lambda E-A \quad B]\left[\begin{array}{ll}
Q & 0 \\
0 & I_{r}
\end{array}\right]\right), \forall \lambda \in \mathbb{C} .
\end{aligned}
$$

Then

$$
\begin{aligned}
& \operatorname{rank}\left[\begin{array}{ccccc}
I_{r_{0}} & 0 & A_{11} & A_{12} & B_{1} \\
0 & 0 & A_{21} & A_{22} & B_{2}
\end{array}\right]
\end{aligned}
$$

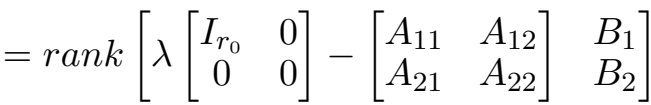

$$
\begin{aligned}
& =\operatorname{rank}\left[\begin{array}{ccc}
\lambda I_{r_{0}}-A_{11} & -A_{12} & B_{1} \\
-A_{21} & -A_{22} & B_{2}
\end{array}\right] \text {. }
\end{aligned}
$$

Therefore

$$
\operatorname{rank}\left[\lambda I_{r_{0}}-A_{11} \quad-A_{12} \quad B_{1}\right]=r_{0}, \quad \forall \lambda \in \mathbb{C} .
$$

Theorem 4. The system (11) is S-controllable if and only if both the conditions (9) and (10) are satisfied.

Proof. The proof follows directly from Proposition 4 .

\section{Illustrating Examples}

In this section, we present some numerical examples which illustrate the effectiveness of the presented theory.

Example 1. [5](page 33),[15](page 8) Consider the circuit system is shown in Figure 1 .

The voltage source $u_{e}$ is the control input. Choose the state variable

$$
x=\left[\begin{array}{llll}
u_{C_{1}} & u_{C_{1}} & I_{1} & I_{2}
\end{array}\right]
$$




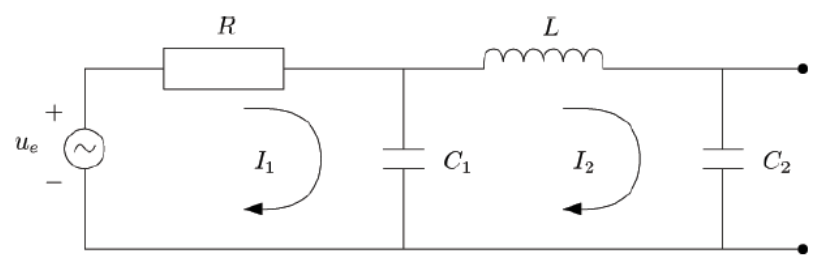

Figure 1: Electrical LCR Circuit

where $u_{C_{1}}$ and $u_{C_{2}}$ are the voltages of $C_{1}, C_{2}$, and the amperage of tile currents flowing over them. According to Kirchoff's second law (Smith, 1966), we may establish the following state equations

$$
\left\{\begin{array}{l}
C_{1} u_{C_{1}}=I_{2} \\
C_{2} u_{\dot{C}_{2}}=I_{1} \\
-L \dot{I}_{1}=-u_{C_{1}}+u_{C_{2}} \\
0=u_{C_{1}}+R I_{2}-u_{e}
\end{array}\right.
$$

The above system of equations can be written as

$$
\begin{aligned}
& {\left[\begin{array}{cccc}
C_{1} & 0 & 0 & 0 \\
0 & C_{2} & 0 & 0 \\
0 & 0 & -L & 0 \\
0 & 0 & 0 & 0
\end{array}\right]\left[\begin{array}{c}
\dot{u}_{C_{1}} \\
\dot{u}_{C_{1}} \\
\dot{I}_{1} \\
\dot{I}_{2}
\end{array}\right] } \\
&=\left[\begin{array}{cccc}
0 & 0 & 0 & 1 \\
0 & 0 & 1 & 0 \\
-1 & 1 & 0 & 0 \\
1 & 0 & 0 & R
\end{array}\right]\left[\begin{array}{c}
u_{C_{1}} \\
u_{C_{1}} \\
I_{1} \\
I_{2}
\end{array}\right] \\
&+\left[\begin{array}{c}
0 \\
0 \\
0 \\
-1
\end{array}\right] u_{e}(t) .
\end{aligned}
$$

In this description equation, we take $C_{1}=C_{2}=1$, $L=1$, and $R=1$. We have $\operatorname{rank} E=3$. By using singular value decomposition of matrix $E$, we get $E=U S V^{\top}$ with

$$
U=\left[\begin{array}{llll}
0 & 0 & 1 & 0 \\
1 & 0 & 0 & 0 \\
0 & 1 & 0 & 0 \\
0 & 0 & 0 & 1
\end{array}\right], S=\left[\begin{array}{llll}
1 & 0 & 0 & 0 \\
0 & 1 & 0 & 0 \\
0 & 0 & 1 & 0 \\
0 & 0 & 0 & 0
\end{array}\right]
$$

and

$$
V=\left[\begin{array}{cccc}
0 & 0 & 1 & 0 \\
1 & 0 & 0 & 0 \\
0 & -1 & 0 & 0 \\
0 & 0 & 0 & 1
\end{array}\right]
$$

By taking $P=U^{-1}$ and $Q=V$, we obtain

$$
P E Q=\left[\begin{array}{cc}
I_{3} & 0 \\
0 & 0
\end{array}\right], \quad P A Q=\left[\begin{array}{cccc}
0 & -1 & 0 & 0 \\
1 & 0 & -1 & 0 \\
0 & 0 & 0 & 1 \\
0 & 0 & 1 & 1
\end{array}\right],
$$

and

$$
P B=\left[\begin{array}{c}
0 \\
0 \\
0 \\
-1
\end{array}\right]
$$

In this system, we have

$$
\operatorname{rank} B_{2}=\operatorname{rank}\left[\begin{array}{lll}
A_{21} & A_{22} & B_{2}
\end{array}\right]=1,
$$

Therefore, by Theorem 11, the circuit network (12) is controllable at infinity.

We have

$$
\operatorname{rank}\left[A_{22} \quad B_{2}\right]=\operatorname{rank}\left[\begin{array}{lll}
A_{21} & A_{22} & B_{2}
\end{array}\right]=1 .
$$

Hence, from Theorem 2, the system (12) is Icontrollable. We have

$$
\operatorname{rank}\left[\lambda I_{3}-A_{11}-A_{12} \quad B_{1}\right]=3, \forall \lambda \in \mathbb{C} .
$$

Hence, from Theorem 3, the system (12) is Ccontrollable.

Also, from (14), (15), and Theorem 4, the circuit network (12) is S-controllable.

Example 2. [5] Consider a simple circuit network as shown in 2, where voltage source $v_{s}(t)$ is the driver (control input), $R, L$, and $C$ stand for the resistor, inductor, and capacity, respectively, as well as their quantities, and their voltages are denoted by $v_{R}(t)$, $v_{L}(t), v_{c}(t)$, respectively. Then from Kirchoff's laws,

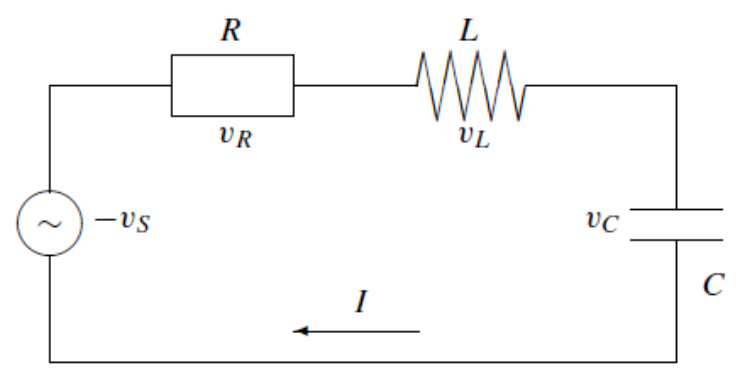

Figure 2: Electrical LCR Circuit

we have the following circuit equations (description equations): 


$$
\begin{aligned}
{\left[\begin{array}{cccc}
L & 0 & 0 & 0 \\
0 & 0 & C & 0 \\
0 & 0 & 0 & 0 \\
0 & 0 & 0 & 0
\end{array}\right]\left[\begin{array}{c}
\dot{I} \\
\dot{v}_{L} \\
\dot{v}_{C} \\
\dot{v}_{R}
\end{array}\right] } \\
=\left[\begin{array}{cccc}
0 & 1 & 0 & 0 \\
\frac{1}{R} & 0 & 0 & 0 \\
-R & 0 & 0 & 1 \\
0 & 1 & 1 & 1
\end{array}\right]\left[\begin{array}{c}
I \\
v_{L} \\
v_{C} \\
v_{R}
\end{array}\right] \\
+\left[\begin{array}{c}
0 \\
0 \\
0 \\
-1
\end{array}\right] v_{s} .
\end{aligned}
$$

We take $L=C=R=1$,

$$
P=I_{4}, \quad \text { and } \quad Q=\left[\begin{array}{cccc}
1 & 0 & 0 & 0 \\
0 & 0 & -1 & 0 \\
0 & 1 & 0 & 0 \\
0 & 0 & 0 & 1
\end{array}\right] .
$$

The system (16) as required for $(6)$ as

$$
\begin{aligned}
& A_{11}=\left[\begin{array}{ll}
0 & 0 \\
1 & 0
\end{array}\right], A_{12}=\left[\begin{array}{cc}
-1 & 0 \\
0 & 0
\end{array}\right], A_{21}= \\
& {\left[\begin{array}{cc}
-1 & 0 \\
0 & 1
\end{array}\right],} \\
& A_{22}=\left[\begin{array}{cc}
0 & 1 \\
-1 & 1
\end{array}\right], B_{1}=\left[\begin{array}{l}
0 \\
0
\end{array}\right] \text { and } B_{2}= \\
& {\left[\begin{array}{c}
0 \\
-1
\end{array}\right] .} \\
& \text { We have } \operatorname{rank} B_{2}=1 \text {, but } \\
& \operatorname{rank}\left[A_{21} \quad A_{22} \quad B_{2}\right]=2,
\end{aligned}
$$

then system (16) is not controllable at infinity, and therefore it is not $\mathrm{C}$-controllable. We have

$$
\operatorname{rank}\left[A_{22} \quad B_{2}\right]=\operatorname{rank}\left[\begin{array}{lll}
A_{21} & A_{22} & B_{2}
\end{array}\right]=2 .
$$

Hence, from Theorem 2, the system (12) is Icontrollable. We have

$$
\operatorname{rank}\left[\lambda I_{2}-A_{11} \quad-A_{12} \quad B_{1}\right]=2, \quad \forall \lambda \in \mathbb{C} .
$$

then, these system is S-controllable.

Example 3. [14] Let the system (11) given by the following matrices

$$
E=\left[\begin{array}{lll}
0 & 1 & 0 \\
0 & 0 & 0 \\
1 & 0 & 0
\end{array}\right], A=\left[\begin{array}{lll}
0 & 0 & 0 \\
0 & 1 & 0 \\
0 & 0 & 0
\end{array}\right], B=\left[\begin{array}{l}
0 \\
1 \\
1
\end{array}\right] .
$$

By taking

$$
P=\left[\begin{array}{ccc}
-1 & 0 & 0 \\
0 & 0 & -1 \\
0 & 1 & 0
\end{array}\right]
$$

and

$$
Q=\left[\begin{array}{ccc}
0 & -1 & 0 \\
-1 & 0 & 0 \\
0 & 0 & 1
\end{array}\right]
$$

we get

$$
\begin{gathered}
P E Q=\left[\begin{array}{ccc}
1 & 0 & 0 \\
0 & 1 & 0 \\
0 & 0 & 0
\end{array}\right], \\
P A Q=\left[\begin{array}{ccc}
0 & 0 & 0 \\
0 & 0 & 0 \\
-1 & 0 & 0
\end{array}\right],
\end{gathered}
$$

and

$$
P B=\left[\begin{array}{c}
0 \\
-1 \\
1
\end{array}\right]
$$

Then

$$
\begin{gathered}
A_{11}=\left[\begin{array}{ll}
0 & 0 \\
0 & 0
\end{array}\right], A_{12}=\left[\begin{array}{l}
0 \\
0
\end{array}\right], A_{21}=\left[\begin{array}{ll}
-1 & 0
\end{array}\right], \\
A_{22}=[0], B_{1}=\left[\begin{array}{c}
0 \\
-1
\end{array}\right] \quad \text { and } B_{2}=[1] .
\end{gathered}
$$

It can be checked that this system is controllable at infinity and I-controllable, but not C-controllable (the condition (10) is also not satisfied for $\lambda=0$ ) and not S-controllable.

Example 4. [15] A general constrained mechanical system can be modeled as

$$
\left\{\begin{array}{l}
\dot{x_{1}}(t)=x_{2}(t) \\
\dot{x_{2}}(t)=C x_{1}(t)+D x_{2}(t)+H^{\top} x_{3}(t)+G u_{1}(t), \\
0=H x_{1}(t)+u_{2}(t)
\end{array}\right.
$$

The details of the above system can be found in Bobinyec et al.[2]. The system (20) can be written in the abstract form (11), if we take

$$
\begin{aligned}
& E=\left[\begin{array}{lll}
I & 0 & 0 \\
0 & I & 0 \\
0 & 0 & 0
\end{array}\right], \quad A=\left[\begin{array}{ccc}
0 & I & 0 \\
C & D & H^{\top} \\
H & 0 & 0
\end{array}\right], \\
& B=\left[\begin{array}{ll}
0 & 0 \\
G & 0 \\
0 & I
\end{array}\right], x(t)=\left[\begin{array}{l}
x_{1}(t) \\
x_{2}(t) \\
x_{3}(t)
\end{array}\right], u(t)=\left[\begin{array}{l}
u_{1}(t) \\
u_{2}(t)
\end{array}\right] .
\end{aligned}
$$

For the numerical purposes, we take the matrices $C, D, H$, and $G$ as follows

$$
\begin{aligned}
& C=\left[\begin{array}{cc}
-2 & 1 \\
1 & -2
\end{array}\right], D=\left[\begin{array}{cc}
0.25 & 0 \\
0 & 0.25
\end{array}\right], \\
& H=\left[\begin{array}{ll}
1 & -1
\end{array}\right], E=\left[\begin{array}{l}
1 \\
1
\end{array}\right] .
\end{aligned}
$$

Then the matrices $E, A$, and $B$ can be rewritten as

$$
E=\left[\begin{array}{lllll}
1 & 0 & 0 & 0 & 0 \\
0 & 1 & 0 & 0 & 0 \\
0 & 0 & 1 & 0 & 0 \\
0 & 0 & 0 & 1 & 0 \\
0 & 0 & 0 & 0 & 0
\end{array}\right],
$$


$A=\left[\begin{array}{ccccc}0 & 0 & 1 & 0 & 0 \\ 0 & 0 & 0 & 1 & 0 \\ -2 & 1 & 0.25 & 0 & 1 \\ 1 & -2 & 0 & 0.25 & -1 \\ 1 & -1 & 0 & 0 & 0\end{array}\right], B=\left[\begin{array}{ll}0 & 0 \\ 0 & 0 \\ 1 & 0 \\ 1 & 0 \\ 0 & 1\end{array}\right]$

By taking

$$
P=\left[\begin{array}{lllll}
0 & 1 & 0 & 0 & 0 \\
0 & 0 & 1 & 0 & 0 \\
0 & 0 & 0 & 1 & 0 \\
1 & 0 & 0 & 0 & 0 \\
0 & 0 & 0 & 0 & 1
\end{array}\right]
$$

and

$$
Q=\left[\begin{array}{lllll}
0 & 0 & 0 & 1 & 0 \\
1 & 0 & 0 & 0 & 0 \\
0 & 1 & 0 & 0 & 0 \\
0 & 0 & 1 & 0 & 0 \\
0 & 0 & 0 & 0 & 1
\end{array}\right]
$$

we get

$$
\begin{aligned}
P E Q & =\left[\begin{array}{ccccc}
1 & 0 & 0 & 0 & 0 \\
0 & 1 & 0 & 0 & 0 \\
0 & 0 & 1 & 0 & 0 \\
0 & 0 & 0 & 1 & 0 \\
0 & 0 & 0 & 0 & 0
\end{array}\right]=\left[\begin{array}{cc}
I_{4} & 0 \\
0 & 0
\end{array}\right], \\
P A Q & =\left[\begin{array}{ccccc}
0 & 0 & 1 & 0 & 0 \\
1 & 0.25 & 0 & -2 & 1 \\
-2 & 0 & 0.25 & 1 & -1 \\
0 & 1 & 0 & 0 & 0 \\
-1 & 0 & 0 & 1 & 0
\end{array}\right],
\end{aligned}
$$

and

$$
P B=\left[\begin{array}{ll}
0 & 0 \\
1 & 0 \\
1 & 0 \\
0 & 0 \\
0 & 1
\end{array}\right]
$$

Then

$$
A_{11}=\left[\begin{array}{cccc}
0 & 0 & 1 & 0 \\
1 & 0.25 & 0 & -2 \\
-2 & 0 & 0.25 & 1 \\
0 & 1 & 0 & 0
\end{array}\right], A_{12}=\left[\begin{array}{c}
0 \\
1 \\
-1 \\
0
\end{array}\right]
$$

$A_{21}=\left[\begin{array}{llll}-1 & 0 & 0 & 1\end{array}\right], A_{22}=[0], B_{1}=\left[\begin{array}{ll}0 & 0 \\ 1 & 0 \\ 1 & 0 \\ 0 & 0\end{array}\right]$,

$B_{2}=\left[\begin{array}{ll}0 & 1\end{array}\right]$,

It can be checked that this system is controllable at infinity, I-controllable, C-controllable, and Scontrollable.

All of the above can be summarized in the following two tables:

\begin{tabular}{|l|c|c|c|c|c|}
\hline & (I) & (II) & (III) & (IV) & (V) \\
\hline Example] & 3 & 1 & 1 & 1 & $3(\forall \lambda \in \mathbb{C})$ \\
\hline Example] & 2 & 1 & 2 & 2 & $2(\forall \lambda \in \mathbb{C})$ \\
\hline Example] & 2 & 1 & 2 & 2 & $1($ for $\lambda=0)$ \\
\hline Example[ & 4 & 1 & 1 & 1 & $4(\forall \lambda \in \mathbb{C})$ \\
\hline
\end{tabular}

(I) $\operatorname{rankE}\left(r_{0}\right)$, (II) $\operatorname{rank} B_{2}$, (III) $\operatorname{rank}\left[\begin{array}{ll}A_{22} & B_{2},\end{array}\right]$, (IV) $\operatorname{rank}\left[\begin{array}{lll}A_{21} & A_{22} & B_{2}\end{array}\right]$, (V) $\operatorname{rank}\left[\lambda I_{r_{0}}-A_{11}-A_{12} \quad B_{1}\right]$.

\begin{tabular}{|c|c|c|c|c|}
\hline & (C1) & (C2) & (C3) & (C4) \\
\hline Example1 & $\checkmark$ & $\checkmark$ & $\checkmark$ & $\checkmark$ \\
\hline Example & & & $\checkmark$ & $\checkmark$ \\
\hline Example 3 & & $\checkmark$ & & $\checkmark$ \\
\hline Example 4 & $\checkmark$ & $\checkmark$ & $\checkmark$ & $\checkmark$ \\
\hline
\end{tabular}

(C1) C-controllable; (C2) controllable at infinity, (C3) S-controllable, (C4) I-controllable

\section{Conclusion}

In this paper, by using an equivalent form, we have given necessary and sufficient conditions for Different concepts related to the controllability of differential-algebraic equations with constant coefficients. The concepts of controllability at infinity, complete controllability, impulse controllability, and strong controllability are described. Finally, we have presented some examples for illustrating the presented theory.

\section{Acknowledgments}

This work was supported by the Laboratory of Fundamental and Applicable Mathematics of Oran (LMFAO) and the Algerian research project: PRFU, no. C00L03ES310120180002 (DGRSDT).

\section{References:}

[1] T. Berger, T. Reis, Controllability of linear differential-algebraic systems a survey, in Surveys in Differential-Algebraic Equations I. Differential-Algebraic Equations Forum, ed. by A. Ilchman, T. Reis (Springer, Berlin, 2013), pp. $1-61$

[2] K. Bobinyec, S. L. Campbell, P. Kunkel, Full order observers for linear DAEs. 50th IEEE conference on decision and control and European control conference (CDC-ECC), Orlando, FL. , 2011, pp. 4011- 4016.

[3] S. L. Campbell, Singular Systems of Differential Equations I, Pitman, San Francisco, CA, 1980. 
[4] M. A. Christodoulou, P. N. Paraskevopoulos, Solvability, controllability, and observability of singular systems, J. Optim. Theory Appl, Vol.45, 1985, pp.53-72.

[5] L. Dai, Singular Control Systems, SpringerVerlag, Berlin, (1989).

[6] D. Cobb, Controllability, observability, and duality in singular systems, IEEE Transactions on Automatic Control, Vol.29 (12), 1984,, pp.10761082.

[7] M. Darouach, M. Boutayeb, Design of Observers for Descriptor Systems, IEEE Trans. on Automatic Control, Vol.40 ,1995, pp.1323-1327.

[8] D. Estévez-Schwarz, U. Feldmann, R. März, S. Sturtzel, C. Tischendorf, Finding beneficial DAE structures in circuit simulation, Technical Report, Institut für Mathematik, Humboldt Universität zu Berlin, Berlin, Germany, 2000, pp.00-7.

[9] M. Hou, Controllability and elimination of impulsive modes in descriptor systems, IEEE Transactions on Automatic Control, Vol.49(10), 2004, pp.1723-1729.

[10] M. Hou et P.C. Müller, Design of a Class of Luenberger Observer for Descriptor Systems, IEEE Trans. on Automatic Control, Vol.40, 1995, pp.133-136.

[11] P. Kunkel, V. L. Mehrmann, Differentialalgebraic equations: analysis and numerical solution, European Mathematical Society, 2006.

[12] D. G. Luenberger, Time Invariant Descriptor System, Automatica, Vol.14, 1978, pp.473-480.

[13] G. Matsaglia and G. P.H. Styan, Equalities and Inequalities for Ranks of Matrices, Linear and Multi-linear Algebra, Vol.2, no.3, 1974, pp.269292.

[14] V. K. Mishra, N. K. Tomar, On complete and strong controllability for rectangular descriptor systems. Circuits Systems and Signal Processing, 2015, pp.1-12.

[15] V. K. Mishra, N. K. Kumar, M. K. Gupta, On Controllability and Normalizability for Linear Descriptor Systems, J. Control Autom. Electr. Syst., Vol.27 (1), 2016, pp.19-28.
[16] L. Pandolfi, On the regulator problem for linear degenerate control systems, J. Optim. Theory Appl., Vol.33, 1981, pp.241-254.

[17] C. C. Pantelides, The consistent initialization of differential-algebraic systems, SIAM J. Sci. Statist. Comput., Vol.9, 1988, pp.213-231.

[18] W. Schiehlen, Multibody Systems Handbook, Springer-Verlag, Heidelberg, 1990.

[19] I. Seufer, Generalized Inverses of DifferentialAlgebraic Operators and their Numerical Discretization, Dissertation, Institut für Mathematik, TU Berlin, Berlin, Germany,2005.

[20] C. Tischendorf, Topological index calculation of differential-algebraic equations in circuit simulation, Surv. Math. Ind., Vol.8, 1999, pp.187-199.

[21] G.Verghese, P. Van Dooren, T. Kailath. Properties of the System Matrices of a Generalized State-Space System, International Journal of Control, Vol.30, 1979, pp.235-243.

[22] P. Wesseling. Principles of Computational Fluid Dynamics, Springer-Verlag, Germany, 2001.

[23] E. L. Yip, R. F. Sincovec, Solvability, Controllability, and Observability of Continuous Descriptor Systems, IEEE Transactions on Automatic Control, Vol. AC-26, 1981, pp.702-707.

[24] Q. Zhang, C. Liu, X. Zhang, Complexity, analysis and control of singular biological systems, Springer, Berlin, Vol.421, 2012.

Contribution of Individual Authors to the Creation of a Scientific Article (Ghostwriting Policy)

The authors equally contributed in the present research, at all stages from the formulation of the problem to the final findings and solution.

Sources of Funding for Research Presented in a Scientific Article or Scientific Article Itself

No funding was received for conducting this study.

\section{Conflict of Interest}

The authors have no conflicts of interest to declare that are relevant to the content of this article.

Creative Commons Attribution License 4.0 (Attribution 4.0 International, CC BY 4.0)

This article is published under the terms of the Creative Commons Attribution License 4.0 https://creativecommons.org/licenses/by/4.0/deed.en 